Breast Cancer Online (www.bco.org) 2004; 7(9),
doi:10.1017/S1470903104001981
O Cambridge University Press
ISSN 1470-9031

\title{
Safety of breast conservation therapy in BRCA1 and BRCA2 cancers
}

\author{
T. E. Alpert ${ }^{1}$, B. G. Haffty ${ }^{2}$ \\ ${ }^{1}$ Department of Radiation Oncology, Upstate Medical University, Syracuse, NY, USA; ${ }^{2}$ Department of \\ Therapeutic Radiology, Yale University School of Medicine, New Haven, CT, USA.
}

\begin{abstract}
For the majority of women with early-stage breast cancer, conservative management with breast conserving surgery plus radiotherapy is widely embraced. However, in patients with germline mutations of BRCA1 and BRCA2, the safety of breast conservation therapy remains controversial. These breast cancer susceptibility genes are associated with an increased risk of second cancers, although the prognosis of BRCA1 or BRCA2 breast cancer is similar to patients with sporadic breast cancer. Preclinical evidence for radiosensitivity has prompted concern for radiation-induced complications in patients with genetic breast cancer. There is limited literature on the safety breast conservation therapy in patients with BRCA1 and BRCA2 mutations. The largest published study reported acute and chronic radiation toxicity results and noted no adverse sequelae in the genetic cohort [1]. Selected published studies demonstrate recurrence rates and overall survival comparable to sporadic controls [1-3]. A series with longer follow-up reported an increase in late second-primary breast cancers, highlighting the need for preventative strategies [4]. Tamoxifen and oophorectomy have a potential role in modifying the rate of second events, and thereby improving the safety of breast conservation therapy.
\end{abstract}

Keywords: BRCA1; BRCA2; Conservation surgery; Prophylaxis

With 20 years of follow-up, breast conservation therapy is an acceptable standard of care for the majority of patients with early-stage breast cancer. The conservative management of breast cancer, by use of wide local excision followed by radiotherapy to the intact breast, has been repeatedly proven to be equivalent to mastectomy for patients with stage I or II disease [5,6]. Five to ten percent of breast cancer patients harbour germline mutations in BRCA1 and BRCA2 and the optimal management of these patients remains controversial [7]. The two major breast cancer predisposition genes were identified

Correspondence to: Bruce G. Haffty, MD, Department of Therapeutic Radiology, Yale University School of Medicine, P.O. Box 208040, New Haven, CT 06520-8040, USA. Email: bruce.haffty@yale.edu; Tel: +1 203785 2959; Fax: +1203785 4622

Publication date 29/09/04

BCO/198/2004/FO in the early 1990s, and they represent the underlying germline mutation in two-thirds of patients with familial breast cancer. BRCA1 and BRCA2 are involved in the DNA repair pathway and this has lead to a basis for hypersensitivity to radiation, as well as the potential for radiation-induced complications [8]. Increased understanding of the biological function of these genes and clinical behaviour of genetic breast cancer has prompted review of conservatively managed patients. Further study is necessary to maximize the safety of breast conservation therapy in patients with BRCA1 or BRCA2 mutations.

\section{Basis for radiosensitivity}

The loci for BRCA1 and BRCA2 are chromosome $17 q 21$ and chromosome 13q12-13, respectively $[9,10]$. Both involved in DNA double-strand break repair 
and function as tumour suppressor genes [11]. The majority of single-gene defects that cause radiosensitivity represent a protein deficiency in either non-homologous end joining or homologous recombination [8]. Mutations in BRCA1 and BRCA2 primarily function by decreasing the rate of homologous recombination and ultimately double-strand break repair [12]. DNA double-strand breaks caused by ionizing radiation could theoretically result in increased cell kill, secondary to defective repair pathways.

Cell-cycle checkpoints are another mechanism of DNA repair that have been implicated in radiosensitivity, particularly in patients with BRCA1 mutations [13]. BRCA1-deficient cells display a defect in the $\mathrm{G}_{2}-\mathrm{M}$ phase checkpoint, and loss of this cell-cycle checkpoint disallows sufficient time for DNA repair [14]. DNA damage that is not repaired may activate apoptosis as a protective mechanism to remove defective cells from a cycling population. While wildtype BRCA1 breast cell lines promote apoptosis in response to ionizing radiation, malignant cells often lack this housekeeping function [15].

The homozygous mutant phenotype of BRCA1, BRCA2, and Rad51 are similar, and murine models have localized BRCA1 and BRCA2 with Rad51 in a postulated common DNA-response pathway [16]. Embryonic cells in mice lacking BRCA2 display hypersensitivity to ionizing radiation [17]. Radiation has been shown to reduce the number of blastocyst and trophoblast cells in homozygous mutants when compared to wild-type, heterozygous, and control embryos. Investigation of mutant mice embryos with homozygous BRCA1 mutation has also documented hypersensitivity to radiation [18].

\section{BRCA1 and BRCA2 breast cancer}

Patients with BRCA1 or BRCA2 mutation have up to an $80 \%$ lifetime risk of breast cancer diagnosis depending on variable penetrance of the gene $[19,20]$. Germline mutation of BRCA1 is also associated with a $20-40 \%$ lifetime risk of developing ovarian cancer [21]. Histopathological features of breast cancer in BRCA1 carriers are more aggressive, with high nuclear grade, aneuploidy, and high proliferation indices [22-25]. Tumours with a medullary component are also common. Oestrogen and progesterone receptors are more often negative when compared with BRCA2 or sporadic counterparts [26]. Initial diagnosis is often at a younger age than patients with sporadic breast cancer, with a median age of 40 years for BRCA1 carriers and 45 years for BRCA2 carriers. Despite these features, BRCA1 and BRCA2 carriers with breast cancer have equivalent survival when compared to age-matched patients with sporadic disease [2].
Patients with BRCA1 and BRCA2 mutations have an increased risk of developing contralateral breast cancer. An early publication by the Breast Cancer Linkage Consortium estimated a $64 \%$ risk of contralateral breast cancer by the age of 70 years in patients with a history of BRCA1 breast cancer [27]. The cumulative risk of ovarian cancer in these patients was $44 \%$ by age 70 years. Women with BRCA2 mutations have a similar risk of developing contralateral breast cancer. There is a lesser risk of ovarian cancer, with a cumulative risk $<10 \%$ by age 70 years [28]. These results should be interpreted with caution, as linkage studies often overestimate risk and several studies of known germline BRCA1 and BRCA2 carriers have demonstrated a lesser increase in the rate of contralateral breast cancer [1-3].

\section{Breast conservation therapy in BRCA1 and BRCA2 carriers}

Early study of familiar breast cancer used positive family history as a surrogate for genetic predisposition. Many of the patients included likely did not harbour germline mutations of the breast cancer susceptibility genes. Identification and cloning of the BRCA1 and BRCA2 germline mutations has prompted genetic testing of patients that previously received breast conservation therapy. Patterns of failure of BRCA1 and BRCA2 carriers have been compared with sporadic patients (Table 1). There are a small number of affected patients and several studies have a relatively short length of median follow-up. Potential sequelae of radiotherapy were analysed in only one series. Survivorship bias is another potential confounding factor when interpreting these results.

Turner et al. published one of the first reports of ipsilateral breast tumour recurrence in patients with BRCA1 and BRCA2 mutations [29]. Using a casecontrol design, the frequency of BRCA1 and BRCA2 mutations was studied in patients who developed an ipsilateral breast recurrence. BRCA1 and BRCA2 testing was performed on all 52 patients with an ipsilateral breast recurrence and the 15 patients in the control group who were $<40$ years of age at diagnosis. Eight patients (15\%) with an ipsilateral breast recurrence had deleterious mutations in BRCA1 or BRCA2. Six (40\%) of these relapsed patients were $<40$ years of age at initial diagnosis compared to 1 of $15(7 \%)$ controls. These results suggest that BRCA1 and BRCA2 mutations are associated with a greater risk of ipsilateral breast recurrence. The median time to recurrence was 7.8 years for patients with BRCA1 or BRCA2 mutation vs. 4.7 years for controls. The majority of recurrences associated with a germline mutation occurred in a different quadrant of the breast and had distinct pathological features. 
Table 1. Patterns of failure after breast conservation therapy.

\begin{tabular}{|c|c|c|c|c|c|c|c|c|c|}
\hline \multirow[b]{2}{*}{ Series } & \multirow{2}{*}{$\begin{array}{l}\text { Number of } \\
\text { patients }\end{array}$} & \multirow{2}{*}{$\begin{array}{l}\text { Follow-up } \\
\text { (years) }\end{array}$} & \multirow{2}{*}{$\begin{array}{l}\text { Number of patients } \\
\text { with BRCA1/2 }\end{array}$} & \multicolumn{3}{|c|}{ Ipsilateral recurrence (\%) } & \multicolumn{3}{|c|}{ Contralateral recurrence (\%) } \\
\hline & & & & Genetic & Sporadic & $P$-value & Genetic & Sporadic & $P$-value \\
\hline Bremer et al. [30] & 110 & 5 & 9 & 29 & 6 & 0.022 & $N^{a}$ & $N R^{a}$ & - \\
\hline Haffty et al. [4] & 127 & 12.7 & 22 & 49 & 21 & 0.007 & 42 & 9 & 0.001 \\
\hline Robson et al. [2] & 305 & 10 & 28 & 22 & 6.9 & 0.25 & 27 & 9.5 & 0.002 \\
\hline $\begin{array}{l}\text { Robson et al. [2]/ } \\
\text { Roberge [3] }\end{array}$ & 505 & 9.7 & 56 & 12 & 8 & 0.68 & 27 & 8 & $<0.001$ \\
\hline Delaloge et al. [31] & 96 & 10 & $\begin{array}{l}37 \text { (BRCA1) } \\
16 \text { (BRCA2) }\end{array}$ & $\begin{array}{r}9 \\
37\end{array}$ & $\begin{array}{l}12 \\
12\end{array}$ & 0.07 & $N R^{a}$ & $N R^{a}$ & - \\
\hline Pierce et al. [1] & 469 & 10 & 170 & 12.5 & 8.6 & 0.55 & 25 & 4 & $<0.0001$ \\
\hline
\end{tabular}

${ }^{a}$ NR: Not reported.

The longer time to recurrence, coupled with change in histology and tumour location suggests that these events may be new primary cancers.

Robson et al. studied breast conservation therapy in Ashkenazi women with the BRCA gene founder mutations (BRCA1 185delAG, BRCA1 5382insC, and BRCA2 617delT) [2]. BRCA gene founder mutations were detected in 28 of the 305 women with retrieved archival tissue. BRCA1 and BRCA2 carriers had a non-significant trend towards increased ipsilateral breast cancer recurrence and decreased overall survival at 5 and 10 years. This trend may be related to the greater likelihood of axillary lymph node involvement and young age in the women with BRCA founder mutations. Involvement of axillary lymph nodes was $48.6 \%$ vs. $32.3 \%(P=0.04)$ for BRCA carriers compared to non-carriers, and the diagnosis at age $<50$ years was $62.9 \%$ vs. $26.2 \%(P<0.001)$, respectively. On univariate as well as multivariate analysis, age was the only factor associated with ipsilateral breast tumour recurrence. The risk of contralateral breast cancer was $14.8 \%$ at 5 years and $27 \%$ at 10 years. This series from Memorial Hospital was later combined with data from McGill University, yielding a total of 56 women with founder mutations [3]. Ipsilateral breast cancer recurrence rates were similar to non-carriers ( $12 \%$ vs. $8 \%$ ). Age $<50$ years at diagnosis was the only significant predictor of metachronous ipsilateral disease $(P=0.002)$. BRCA1 mutation was an independent predictor of breast cancer mortality on multivariate analysis, but only for women who did not receive chemotherapy. BRCA2 mutations had no impact on breast cancer specific survival. Again, BRCA1 and BRCA2 carriers had an elevated risk of contralateral breast cancer at a median follow-up of 9.7 years $(27 \%$ vs. $8 \%, P<0.001)$.

Haffty et al. studied breast conservation therapy in germline mutation patients with early-onset breast cancer and compared outcomes to patients with sporadic breast cancer [4]. One hundred and twentyseven women diagnosed with breast cancer at age
42 years or younger agreed to undergo genetic testing, and 22 were found to have BRCA1 or BRCA2 mutations. Patients in the genetic group were younger than sporadic patients, and this difference was significant on multivariate analysis. Adjuvant tamoxifen or oophorectomy were not used in any of the carriers of BRCA1 or BRCA2 mutations. With a median follow-up of 12.7 years, the genetic group had a higher rate of contralateral ( $42 \%$ vs. $9 \%, P=0.001)$ as well as ipsilateral breast events (49\% vs. $21 \%, P=0.007)$. The rate of contralateral and ipsilateral events was much higher than previously reported series and may be attributable to the younger age at diagnosis, longer duration of follow-up, and lack of adjuvant oophorectomy or tamoxifen. The proportion of relapse-free BRCA1 and BRCA2 carriers was similar to non-carriers at 5 years, and then progressively declined with time. Nine of the 11 ipsilateral breast recurrences were classified as second primary tumours, based on a difference in tumour location $(n=7)$ and/or histology $(n=8)$. All second events in the BRCA1 and BRCA2 carriers were successfully salvaged. Bremer et al. recently confirmed the increased risk of developing ipsilateral second primaries in BRCA1 and BRCA2 carriers and extended this concern to patients with bilateral breast cancer [30]. The high incidence of ipsilateral breast recurrence is likely to be reduced to an acceptable level with the use of tamoxifen or oophorectomy.

Breast cancer diagnosis at a young age may be associated with a greater genetic penetrance of germline mutations. A recent study from the Institut Gustave Roussy found age to be the major predictor of relapse in conservatively managed patients with BRCA1 and BRCA2 or non-BRCA familial breast cancer [31]. Of the 96 patients, 37 had BRCA1 mutations and 17 had BRCA2 mutations. On multivariate analysis, diagnosis at age $<40$ years was the only predictor of local failure. The 10-year local relapse-free survival was $73 \%$ for age $<40$ years vs. $91 \%$ for age $>40$ years $(P=0.002)$. 
Using a multi-institutional collaborative group database, Pierce et al. analysed breast conservation therapy in 71 women with BRCA1 or BRCA2 mutation matched 1: 3 with sporadic breast cancer patients [1]. This is the largest reported study of patients with BRCA1 and BRCA2 mutations and the only study to specifically compare complications of radiotherapy. Using acute Radiation Therapy Oncology Group (RTOG) scoring, the incidence of acute lung and skin toxicity was reported [32]. The incidence and severity of breast pain was also documented. Chronic radiation complications were scored using the RTOG/ EORTC (European Organization for Research and Treatment of Cancer) late radiation morbidity scoring scheme [32]. The cohorts were well matched with respect to age at diagnosis, axillary lymph node status, prophylactic measures, and radiotherapy treatment fields and dose. Specifics of radiotherapy treatment were an obvious omission in other series. At 5 years, the risk of developing a contralateral breast cancer was $20 \%$ in BRCA1 and BRCA2 carriers vs. $2 \%$ in the sporadic cohort $(P<0.0001)$. Local tumour control was comparable in BRCA1 and BRCA2 carriers vs. the sporadic cohort, $98 \%$ vs. $96 \%$ at 5 years. The median time to breast recurrence for the three patients in the genetic group was 8.2 years. This prolonged time interval is consistent with other series of genetic breast cancer patients and suggests the need for lengthy follow-up. Ten-year outcomes were recently presented, and relapse free and overall survival did not differ by cohort [33]. In the genetic cohort, the risk of contralateral breast cancer remained elevated $(25 \%$ vs. $4 \%, P=0.0001)$, while ipsilateral breast cancer recurrence was not significantly increased ( $12.5 \%$ vs. $8.6 \%$ ). There was no difference in acute lung or skin toxicity, or breast pain. Only $1 \%$ of genetic cases and $3 \%$ of sporadic cases developed confluent most desquamation (grade 3 toxicity), and there were no cases of ulceration or necrosis (grade 4 toxicity). No acute pulmonary toxicity occurred in $97 \%$ of the genetic cohort, and the remaining $3 \%$ developed only grade 1 toxicity (mild dry cough or dyspnoea on exertion). The number of patients who reported breast pain as well as number of patients who required analgesia were similar. There was no significant difference in the rate of skin, subcutaneous tissue, lung, or bone complications using the late RTOG/EORTC scoring scheme. While retrospective data may underreport toxicity scoring, BRCA1 and BRCA2 mutations were not associated with any measured toxicities.

\section{Risk reduction strategies}

Most risk reduction strategies have focused on primary prevention, but prophylactic strategies should also be considered at the time of breast cancer diagnosis. Oophorectomy and tamoxifen offer similar risk reduction for breast cancer patients with germline mutations. Women with BRCA1 or BRCA2 mutations who underwent prophylactic oophorectomy to reduce the risk of ovarian cancer were found to have a decreased incidence of breast cancer. Tamoxifen has been shown to reduce both the risk of breast cancer in high-risk women and the risk of contralateral breast cancer in patients with ipsilateral breast cancer $[34,35]$.

Rebbeck et al. studied the risk of breast cancer in 43 BRCA1 carriers with no history of breast or ovarian cancer who underwent prophylactic bilateral oophorectomy [36]. When these patients were compared to BRCA1 controls who did not undergo oophorectomy, there was a significant reduction in breast cancer risk (hazard ratio, 0.53). The magnitude of the benefit increased with longer follow-up, with a hazard ratio of 0.28 for patients followed 5 to 10 years, and 0.33 for patients with $>10$ years follow-up. The Prevention and Observation of Surgical End Points Study Group (PROSE) identified 551 women with BRCA1 or BRCA2 germline mutations and reported the incidence of ovarian and breast cancer in women that had undergone prophylactic oophorectomy vs. matched controls [37]. With a median follow-up of 8 years, two women developed papillary serous peritoneal carcinoma after oophorectomy and 58 controls were diagnosed with ovarian cancer. After the exclusion of the six women who were diagnosed with cancer at surgery, oophorectomy reduced the risk of ovarian cancer by $96 \%$. Of the 241 women with no history of breast cancer or prophylactic mastectomy, oophorectomy also reduced the incidence of breast cancer $(42.3 \%$ vs. $21.2 \%$, hazard ratio 0.47 ).

A prospective study of the risk of gynaecological cancer and breast cancer from Memorial SloanKettering Cancer Center identified 170 BRCA1 or BRCA2 carriers who chose to undergo surveillance or prophylactic oophorectomy [38]. With a median follow-up of only 24 months, this prospective study supports an early reduction in breast and ovarian cancer risk with prophylactic oophorectomy. Among the surveillance group of 72 patients, 8 patients developed breast cancer, 4 ovarian cancer; and 1 peritoneal cancer. Of the 98 women who opted for prophylactic oophorectomy, 3 were later diagnosed with breast cancer and one patient developed peritoneal cancer.

Tamoxifen has been extensively studied by the National Surgical Adjuvant Breast and Bowel Project (NSABP) in women at high risk for invasive breast cancer. An analysis of the NSABP data identified 19 BRCA1 and BRCA2 mutations in the 288 women who developed breast cancer [39]. Five of the eight women 
with BRCA1 mutations had received tamoxifen vs. three of eleven women with BRCA2 mutations. This represented a $62 \%$ reduction in breast cancer incidence for BRCA2 carriers, but no benefit for tamoxifen in BRCA1 carriers. However, the data set was small and had low power to detect a protective effect.

Narod et al. studied tamoxifen and the risk of contralateral breast cancer in a study of BRCA1 and BRCA2 carriers [40]. This collaborative case-control study compared women with bilateral breast cancer and women with unilateral breast cancer. A higher percentage of BRCA2 carriers used tamoxifen than BRCA1 carriers, $33 \%$ vs. $13 \%$, respectively. Tamoxifen protected against contralateral breast cancer, with an odds ratio of 0.38 for BRCA1 carriers and 0.63 for BRCA2 carriers. BRCA1 patients are more likely to have oestrogen receptor-negative tumours, likely requiring a larger sample size to detect a benefit for tamoxifen in BRCA1 carriers. This study also noted a reduction in contralateral breast cancer in patients who received oophorectomy. The odds ratio was 0.42 , a similar magnitude to the reduction in contralateral breast cancer demonstrated with tamoxifen.

Both oophorectomy and tamoxifen have not been widely used in studies of conservatively managed breast cancer patients with BRCA1 and BRCA2 mutations. Their potential benefits must be weighed against the possible complications of premature menopause following oophorectomy and the side effects of tamoxifen. The use of selective oestrogen receptor modifiers to prevent second events is an area for further study.

\section{Conclusions}

The conservative management of breast cancer in patients with BRCA1 and BRCA2 germline mutations highlights several complex issues. Theoretical concerns for radiation-induced complications have not been demonstrated in the limited published data. Lengthy follow-up is necessary as there is a prolonged interval to relapse in patients with BRCA1 and BRCA2 mutations. Series with relatively short follow-up may underestimate the rate of ipsilateral breast recurrence and the incidence of contralateral breast cancer. The high rate of successful surgical salvage of in-breast relapse is promising. Risk reduction strategies to prevent second events warrant further study, as they are likely to improve the safety of breast conservation therapy.

\section{References}

1. Pierce LJ, Strawderman M, Narod SA, et al. Effect of radiotherapy after breast-conserving treatment in women with breast cancer and germline BRCA1/2 mutations. J Clin Oncol 2000; 18: 3360-3369.
2. Robson M, Levin D, Federici J, et al. Breast conservation therapy for invasive breast cancer in Ashkenazi women with BRCA gene founder mutations. J Nat/ Cancer Inst 1999; 91: 2112-2117.

3. Robson ME, Chappuis PO, Satagopan J, Wong N, Boyd J, Goffin JR, Hudis C, Roberge D, Norton L, Begin LR, Offit K, Foulkes WD. A combined analysis of outcome following breast cancer: differences in survival based on BRCA1/ BRCA2 mutation status and administration of adjuvant treatment. Breast Cancer Res 2004; 6(1): R8-R17.

4. Haffty BG, Harrold E, Knan AJ, et al. Outcome of conservatively managed early-onset breast cancer by BRCA1/2 status. Lancet 2002; 359: 1471-1477.

5. Fisher B, Anderson S, Bryant J, et al. Twenty-year followup of a randomized trial comparing total mastectomy, lumpectomy, and lumpectomy plus irradiation for the treatment of invasive breast cancer. New Engl J Med 2002; 347: 1233-1241.

6. Veronesi U, Cascinelli N, Mariani L, et al. Twenty-year follow-up of a randomized study comparing breastconserving surgery with radical mastectomy for early breast cancer. New Engl J Med 2002; 347: 1227-1232.

7. Claus EB, Schildkrant JM, Thompson WD, et al. The genetic attributable risk of breast and ovarian cancer. Cancer 1996; 77: 2318-2324.

8. Xia F, Powell S. The molecular basis of radiosensitivity and chemosensitivity in the treatment of breast cancer. Semin Radiat Oncol 2002; 12: 296-304.

9. Hall JM, Lee MK, Newman B, et al. Linkage of early onset familial breast cancer to chromosome 17q21. Science 1990; 250: 1684-1689.

10. Wooster R, Neuhausen SL, Mangion J, et al. Localization of a breast cancer susceptibility gene, BRCA2, to chromosome 13q12-13. Science 1994; 265: 2088-2090.

11. Buchholz TA, Wazer DE. Molecular biology and genetics of breast cancer development: a clinical perspective. Semin Radiat Oncol 2002: 12: 285-295.

12. Zhong Q, Chen CF, Li S, et al. Association of BRCA1 with the hRad50-hMre11-p95 complex and the DNA damage response. Science 1999; 285: 747-750.

13. Xu B, Kim S, Kastan MB. Involvement of BRCA1 in S-phase and G(2)-phase checkpoints after ionizing irradiation. Mol Cell Biol 2001; 21: 3445-3450.

14. Xu X, Weaver Z, Linke SP, et al. Centrosome amplification and a defective G2-M cell cycle checkpoint induce genetic instability in BRCA1 exon 11 isoform-deficient cells. Mol Cell 1999; 3: 389-395.

15. Thangaraju M, Kaufmann SH, Couch FJ. BRCA1 facilitates stress-induced apoptosis in breast and ovarian cancer cell lines. J Biol Chem 2000; 32: 33487-33496.

16. Chen JJ, Silver D, Cantor S, Livingston DM, Scully R. BRCA1, BRCA2, and Rad51 operate in a common DNA damage response pathway. Cancer Res 1999; 59 (Suppl): 1752-1756.

17. Sharan SK, Morimatsu M, Albrecht U, et al. Embryonic lethality and radiation hypersensitivity mediated by Rad51 in mice lacking Brca2. Nature 1997; 386: 804-810.

18. Shen S, Weaver Z, Xu X, et al. A targeted disruption of the murine BRCA1 gene causes $\gamma$-irradiation hypersensitivity and genetic instability. Oncogene 1998; 17: 3115-3124.

19. Porter DE, Cohen BB, Wallace MR, et al. Breast cancer incidence, penetrance, and survival in probable carriers of BRCA1 gene mutation in families linked to BRCA1 
on chromosome 17q12-21. Br J Surg 1994; 81: 1512-1515.

20. Ford D, Easton DF, Stratton M, et al. Genetic heterogeneity and penetrance analysis of the BRCA1 and BRCA2 genes in breast cancer families: The Breast Cancer Linkage Consortium. Am J Hum Genet 1998; 62: 676-689.

21. Easton DF, Ford D, Bishop DT. Breast and ovarian cancer incidence in BRCA1-mutation carriers: Breast Cancer Linkage Consortium. Am J Hum Genet 1995; 56: 265-271.

22. Lynch HT, Marcus JN, Watson P, Page D. Distinctive clinical pathologic features in BRCA1 linked hereditary breast cancer. Proc ASCO 1994; 13: 56 [Abstract 27].

23. Jacquemier J, Eisinger F, Birnbaum D, et al. Histoprognostic grade in BRCA1-associated breast cancer. Lancet 1995; 345: 1503.

24. Marcus JN, Watson P, Page DL, et al. Hereditary breast cancer: pathobiology, prognosis, and BRCA1 and BRCA2 gene linkage. Cancer 1996; 77: 697-709.

25. Breast Cancer Linkage Consortium. Pathology of familial breast cancer: differences in carriers of BRCA1 or BRCA2 mutations and sporadic cases. Lancet 1997; 349: 1505-1510.

26. Verhoog LC, Brekelmans CTM, Seynaeve C, et al. Survival and tumor characteristics of breast-cancer patients with germline mutations of BRCA1. Lancet 1998; 351: 316-321.

27. Ford D, Easton DF, Bishop DT, et al. Risks of cancer in BRCA1-mutation carriers. Lancet 1994; 343: 692-695.

28. Ford D, Easton DF. The genetics of breast an ovarian cancer. Br J Cancer 1995; 72: 805-812.

29. Turner BC, Harold E, Matloff E, et al. BRCA1/BRCA2 germline mutations in locally recurrent breast cancer patients after lumpectomy and radiation therapy: implications for breast-conserving management in patients with BRCA1/BRCA2 mutations. J Clin Oncol 1999; 17: 3017-3024.

30. Bremer M, Doerk T, Sohn C, Karstens JH. Local relapse after postoperative radiotherapy in patients with bilateral breast cancer by BRCA1/2 status. Proc ASCO 2003; 22: 11 [Abstract 42].
31. Delaloge S, Kloos I, Ariane D, et al. Young age is the major predictor of local relapse among conservatively treated BRCA1-, BRCA2-, or non BRCA-linked hereditary breast cancer (BC). Proc ASCO 2003; 22: 11 [Abstract 41].

32. Cox JD, Stetz J, Pajak TF. Toxicity criteria of the Radiation Oncology Group (RTOG) and the European Organization for Research and Treatment of Cancer (EORTC). Int J Radiat Oncol Biol Phys 1995; 31: 1341-1346.

33. Pierce L, Levin A, Rebbeck T, et al. Ten-year outcome of breast-conserving surgery (BCS) and radiotherapy (RT) in women with breast cancer $(\mathrm{BC})$ and germline BRCA 1/2 mutations: results from an international collaboration. 26th Annual San Antonio Breast Cancer Symposium, San Antonio, TX, 3-6 December 2003.

34. Fisher B, Constantino JP, Wickerham DL, et al. Tamoxifen for prevention of breast cancer; report of the National Surgical Adjuvant Breast and Bowel Project P1 study. J Nat/ Cancer Inst 1998; 90: 1371-1388.

35. Early Breast Cancer Trialists' Collaborative Group. Tamoxifen for early breast cancer: an overview of the randomized trials. Lancet 1998; 351: 151-167.

36. Rebbeck TR, Levin AM, Eisen A, et al. Breast cancer risk after bilateral prophylactic oophorectomy in BRCA1 mutation carriers. J Natl Cancer Inst 1999; 91: 1475-1479.

37. Rebbeck TR, Lynch HT, Neuhausen SL, et al. Prophylactic oophorectomy in carriers of BRCA1 or BRCA2 mutations. New Engl J Med 2002; 346: 1616-1622.

38. Kauff ND, Satagopan JM, Robson ME, et al. Risk-reducing salpingo-oophorectomy in women with a BRCA1 or BRCA2 mutation. New Engl J Med 2002; 346: 1609-1615.

39. King M, Wieand S, Hale K, et al. Tamoxifen and breast cancer incidence among women with inherited mutations in BRCA1 and BRCA2. J Am Med Assoc 2001; 286: 2251-2256.

40. Narod SA, Brunet J, Ghadirian P, et al. Tamoxifen and risk of contralateral breast cancer in BRCA1 and BRCA2 mutation carriers: a case control study. Lancet 2000; 356: 1876-1881. 